\title{
Experiences from Cervical Cancer Screening Program Conducted at Low-Resource Areas in Telangana
}

\author{
Sravya Thumoju ${ }^{1,3, *}$, Sireesha Divyakolu ${ }^{1,5}$, Triveni Bhopal' ${ }^{2}$, Santoshini Gowrishetty ${ }^{1,5}$, Sayeda S Fatima ${ }^{1,6}$, \\ Yog Raj Ahuja' ${ }^{1}$, Vasavi Mohan ${ }^{1,3,4}$
}

\section{Sravya Thumoju ${ }^{1,3, *}$, Sireesha Divyakolu, ${ }^{1,5}$, Triveni Bhopal', Santoshini Gowrishetty ${ }^{1,5}$, Sayeda S Fatima ${ }^{1,6}$, Yog Raj Ahuja' ${ }^{1}$, Vasavi Mohan ${ }^{1,3,4}$}

'Department of Genetics and Molecular Medicine, Vasavi Medical and Research Centre, Lakdikapul, Hyderabad, Telangana, INDIA.

${ }^{2}$ Department of Pathology, MNJ Institute of Oncology Regional

Cancer Centre, Lakdikapul, Hyderabad, Telangana, INDIA.

${ }^{3}$ Department of Genetics and Molecular Medicine, Kamineni Hospitals, L.B Nagar, Hyderabad, Telangana, INDIA. ${ }^{4}$ Hansgene Cancer Foundation, Khairatabad, Hyderabad, Telangana, INDIA.

${ }^{5}$ Gleneagles Global Hospital, Lakdikapool, Hyderabad, Telangana, INDIA.

${ }^{6}$ Dr. Remedies Labs, Punjagutta, Hyderabad, Telangana, INDIA.

\section{Correspondence}

\section{Ms. SravyaThumoju}

Department of Genetics and Molecular Medicine, Vasavi Medical and Research Centre, Lakdikapul, Hyderabad-500 004, Telangana, INDIA.

Mobile no: +91-9963138782

Email: sravya.thumoju@gmail.com

History

- Submission Date: 17-10-2017

- Revised Date: 26-02-2018

- Accepted Date: 04-08-2018

DOI : 10.5530/ijmedph.2018.3.24

Article Available online

http://www.ijmedph.org/v8/i3

\section{Copyright}

(c) 2018 Phcog.Net. This is an openaccess article distributed under the terms of the Creative Commons Attribution 4.0 International license.

\begin{abstract}
Background: Cancer of the uterine cervix is preventable and early detection is key to its cure. Screening methods for cervical cancer are available, however, the centres offering to screen are inadequate. The main obstacle for the success of screening efforts in women is the lack of awareness, hence a stigma about the screening procedure and the disease, not just in rural areas but also in a large group of the urban and semi-urban population. The purpose of this manuscript is to share our experiences during health camps conducted for screening women for cervical cancer, strategies adopted, leading to better compliance and complete evaluation in these women. Design and Methods: Health camps were organized for women's screening and we observed a steady improvement in their participation over a period of time, at rural and peri-urban areas close to the Hyderabad. Creating awareness, training para-medical staff, involving local voluntary bodies for conducting Pap testing and HPV subtyping, gave us optimal leads to manage these patients appropriately and recommend a suitable follow-up. Results and Conclusion: Taking a lead from studies like this and others, conducting national screening programs along with counselling for awareness of such tests and their implications to maintain good health, the burden of cervical cancer can be reduced in India.
\end{abstract}

Key words: Uterine cervix, High risk HPV sub-types, Cervical screening, Cervical Cancer.

\section{INTRODUCTION}

Worldwide women are more prone to breast and uterine cervix cancers because of the constant hormonal fluctuations occurring throughout the reproductive life cycle. Cervical cancer has become the leading cause for mortality in women worldwide, especially in developing countries, estimated to be the fourth common cancer among women (Globocan, 2012). ${ }^{1}$ In India, urban areas like Thiruvananthapuram, Mumbai and Delhi, breast cancer leads in the number of cases compared to peri-urban areas like Aizwal, Guwahati and Barshi, where cervical cancer is most common. ${ }^{2}$ Almost $70 \%$ of the global burden falls on developing countries; more than one-fifth of all new cancer cases are diagnosed in India according to WHO-2013. Chennai Cancer registry recorded a $15 \%$ of cervix cancer during the year 2006-2008 as compared to the other cancers. ${ }^{3}$ Hospital-based evidence from our study during 2012-2013, recorded a total of 420 cervical cancer cases from a major centres in Hyderabad. HPV is recognized to be an important etiological agent of cervical cancer. In Eastern India, 9.9\% of HPV prevalence was seen in women without cervical cancer and more HPV 18 sub-type as compared to HPV 16 was noted. ${ }^{4}$ While lack of basic facilities for testing is contribu- tory, a lack of follow-up is also one of the drawbacks in our country especially in low-resource areas. ${ }^{5}$ Despite having a higher mortality rate in India, till today there are few national screening programs in place for cervical screening of women. There was a $31 \%$ reduction in the mortality rate after cervical cancer screening by preliminary health workers in Mumbai. ${ }^{6}$ In Kerala, screening programs met with low rates of compliance (6.9\%), due to many factors like stigma associated with the screening process, fear of procedure, lack of awareness, etc. ${ }^{7}$ The difficulties in conducting a population-based cervical cancer screening program are attributed to non-availability of expert cytologists to screen samples, inadequate infrastructure for conducting screening for women at low-resource areas and no proper laboratory facilities for testing. ${ }^{8}$ In addition to this, lack of awareness in women about the necessity for cervical screening makes non-compliance a big hindrance to the effort put into organizing screening camps. It is our aim through this paper, to share our experiences for cervical screening where we identified and used appropriate strategies during our camps to ensure close to $100 \%$ compliance.

A general screening of cervix to prevent cancer, should be performed in sexually active women, above the 
age of 25 years as per our earlier study, where it would involve visual examination followed by VIA alone or VIA combined with a pap smear for cytological evaluation of the ecto- and endo-cervical cells for erosive lesions, any signs of malignancy or early disease. ${ }^{9}$ Further it is advisable that cases showing any abnormalities in cervix be tested for presence of high-risk HPV infection in order to undertake appropriate intervention post-testing. Facilities for molecular analysis for HPV detection and typing are restricted to few city-based laboratories and this has become a major drawback for testing for HPV sub-types prevalent in our population, with associated risk for cervical cancer.

Cervical cancer incidences are gradually increasing; the potential risk factor for this type of cancer is the Human Papilloma Virus (HPV), commonly associated with genital warts. More than 100 different sub-types of HPV with distinguished variations in its genetic and oncogenic potential are known. The sub-types which precisely affect the anogenital tract are reported to be HPV sub-types $16,18,31,33,35,39,45,51,52$, $56,58,66$ and $69 .^{10}$

The other risk factors like smoking, alcohol consumption, unsafe sexual practices and lack of basic hygiene might play a major role in development of cervical cancer. Men who have multiple sexual partners or who are carriers of HPV DNA are vectors of high-risk HPV subtypes and place their partner at an increased risk of developing cancer of the cervix. However those subtypes specific to our population have still not been addressed (subject of another manuscript). Commercial sex workers are an important reservoir of high-risk HPVs. Other risk factors that have not been assessed here may be stress, heavy work load leading to irregular hormonal changes etc. in women. ${ }^{11}$

\section{METHODOLOGY AND RESULTS}

This work was carried out at Vasavi Medical and Research Centre, Lakdikapul, Hyderabad, India and Hansgene Cancer Foundation, Khairatabad, Hyderabad, India during the period of September 2011- August 2014. This study was approved by Vasavi Institutional Ethics Committee, Vasavi Hospital, Hyderabad.

\section{Screening}

Cervical cancer screening can be done by various methods. Visual inspection of cervix (VIA - poorly used but efficient testing method), Pap test (Papanicolaou test) and HPV sub-typing are recommended. A Pap test is a screening test of ecto- and endo-cervical cells for potential pathological changes. Based on presence of cervical erosions/lesions or suspicious cells on cytology, further testing is done by colposcopic biopsy, X ray, CT scan, MRI and PET scan.

\section{Pre-camp arrangements}

With the help of local organizations (Lions Club, Shakti Shifa Health foundation and Family Planning Association of India) health camps were conducted. A site visit prior to conducting the camp helped us check out the place for infrastructure and facilities available. Depending on the possible strength of women likely to attend the camp, sufficient testing material was carried to the camp. In addition to other pre-camp procedures, a local person on-site was trained on giving a pre-camp lecture to educate the women to ensure participation and to derive the benefit of screening.

\section{Requirements}

Speculums need to be sterilized in an autoclave, we can also use disposable speculums; butterfly spatula or cytobrush for collecting cervix cells from the ecto- and endo-cervical regions; fissy spray (company name) to fix tissue cells on slide; labeled slides for collecting pap samples from various patients for pap staining, labeled screw cap tubes for collecting scrapings/ cells for DNA isolation for molecular analysis; saline solution for collecting tissue/ cervix scrapings sample for DNA isolation; slide holders/tray for holding slides; hand gloves; lamp for visual examination, ethanol to sterilize equipment and cotton; well-designed proformas to collect patient's medical and personal details. The minimum safety precaution materials like gloves, betadine, spirit and masks were also carried.

\section{Pre-Camp Counselling of participants (One on One)/training of Para-medical staff}

The women were counselled about their health and hygiene, the importance of regular screening tests and how it reduces the chances of their disease progression and facilitates easy management. We organized pre-visits at the scheduled camp area to educate people on purpose of attending the camp and the screening and also to educate para-medical and other staff on appropriate practices for suitable sampling and patient interaction. We used models available in the healthcare centre or our team carried them to describe mode of testing and necessity for cervical screening of women to avoid cancer risk due to late detection, the benefit of early pre-cancers detection and their treatment and management of the disease. Medical and para-medical staff at local healthcare centres were involved and trained on aspects of counselling, data collection, appropriate collection of Pap smear for cytology and post-test counselling. They were also sensitized to follow certain protocols for successful conductance of these cervical cancer screening camps. Such a method increased the volunteerism from local staff and compliance of subjects for cervical screening. The ideal time for collection of a pap smear would be two weeks after the menstrual flow which is the proliferative phase, while in menopausal women, it can be sampled at any time. A total of 16 health camps were conducted during the year 2011 to 2014 at different rural areas of Telangana (Padmashalisangam, Koti Women's college, Kadthal-I and II, Kothapet camp, Peddapalli, Vikarabad, Family planning association -I and II, Shaktishifa health foundation-I,II and III, Vasavi Hospital, Lions club, Vavilalapalli and Achampet ). For initial few camps, we were faced with issues of non-compliance of a number participants (5-20\% compliance) who visited the screening camp (during health camp women expected to receive free medicines (multi vitamin pills) rather than getting cervical screening done). Subsequently, we started counselling the participants before sampling in groups where we showed a presentation representing how a women's reproductive system functions and explained about the importance of cervical screening to prevent the cancer risk, detection in early stages and the preventable costs thereof. Where necessary, even one-to-one counseling was also given to ensure participation eventually increasing the compliance rates to $80-100 \%$.

Proformas were filled in before collecting cervical sample from the patient. Patient's details such as name, menstrual history, age, lifestyle and dietary habits, clinical history, any health complications like hypertension, diabetes etc were noted down along with details of visual inspection while collecting details. After taking all the details, participant consent was taken before sampling.

\section{Organizing screening camps}

Meticulous planning and implementation, with proper cleaning and washing area for the equipment is a must for every screening camp apart from other things. Counselling while collecting personal and medical details was done and patient was made aware of implications of the testing results, good hygiene maintenance, etc. VIA can be performed prior to collection of a pap smear to identify whitened areas indicative of probable abnormal tissue. Sample collection of cervix tissues was done by certified gynecologist/medical officer on-site who examine the cervix under lamp light; with the help of a speculum, cervical cells were collected either with the help of butterfly spatula or a cytobrush after clearing the 
mucus with a cotton swab so that it did not interfere with sample collection and spreading of cells on the slide. The ecto- and endo cervical cells were spread on a slide evenly for Pap smear test and sprayed with a fixative for fixing the cells on the slide. In addition, a cervical smear was transferred to a screw-cap tube containing $4 \mathrm{ml}$ saline solution and kept till the further processing for DNA isolation for HPV detection work.

\section{Post-camp process}

The slides were stained with conventional pap staining procedure and sent to a certified pathologist for pap staining and screening; based on the morphology, the pap cytology and infection status was reported.

Reports were given to local medical officers and the follow-up of abnormal cases was done by re-calling the subjects for colposcopy examination by our Gynecologist for further study.

\section{Important infections on Pap smear}

Several infections of the vagina and cervix can be identified as a part of the routine Pap smear. In Trichomonas vaginalis infection we can see numerous cytoplasmic reddish granules and occasional nuclei were seen; in Candida infection, filamentous slender structures, in Gardnerella vaginalis, small and short Gram-variant coccobacilli clustered onto the squamous epithelial cells. Clue cells are identified in a pap smear by the presence of epithelial cells covered with gram variable bacilli indicating bacterial vaginosis, this appears purplish as compared to pinkish-red epithelial cells. ${ }^{12-13}$

Cervix cells collected in screw-cap tubes were processed for DNA isolation. Subsequently PCR was performed to detect presence of HPV in the samples and those positive for HPV DNA were subjected to high-risk HPV sub-typing by multiplex PCR. Later, we correlated the demographic details of the patients with high-risk HPV, co-infections and pap cytology.

\section{Abnormal cases follow-up}

Cases with abnormal pap cytology were sent for colposcopic examination and biopsy as recommended by gynecologist, if the Pap test showed a normal cytology those women were referred for follow-up Pap test in 3-5 years. Cases of abnormal pap cytology and/or positive HPV with high risk HPV sub-type cases and cases with cervical infections were called for gynecological consultation and appropriate management / medication to arrest further progression of cervical disease.

For initial few camps we did VIA prior to collection of the pap sample, though it was slightly time consuming procedure. Out of 530 women who participated in the screening program 317 were samples with normal cytology, 139 were infectious and 74 (13.9\%) samples with abnormal pap cytology; these are population-based cervical screening numbers. Among cases with infection, we mostly observed bacterial vaginosis or candida. Trichomonas was seen in two HIV cases that we chanceencountered. We did not perform VIA for all the samples as we observed that it correlated with pap cytology and direct visual inspection. Hospital based screening was done by our own group prior to this study where again it was found that pap cytology correlated with VIA examination ${ }^{14}$ $\sim 2 \%$ of all our cervical samples tested positive for high-risk HPV infection. This included one of the HIV positive patients. These were followed up with colposcopy and biopsy where essential to rule out malignancy; in cases where suggested, hysterectomy was performed. There were other samples that tested positive for low-risk HPVs.

\section{DISCUSSION}

The main obstacle in successful cervical cancer screening program is the stigma attached to a pelvic examination in women. In our experience, when we started women's health camps for cervical screening in 2011, we observed very poor compliance from the local women to participate and get tested. After few initial camps, we changed our approach - we con- ducted a general awareness talk with the help of the local health officer about cervical screening at a pre-camp visit. On-site personnel training was also given during this visit. We then adopted the strategy of oneto-one counselling of each woman who visited the camp in their native language, on importance of testing to avoid future risk for cancer, safety of the procedure; eventually, the Pap smear was collected in addition to collecting cervical smear for DNA isolation and HPV testing. Compliance rates drastically improved from $\sim 10 \%$ to nearly $100 \%$ participation for screening by the subjects after counselling the participants about the importance of screening. (The occasional cases where the women did not undergo screening were when she had undergone a hysterectomy or had attended camp during her menstrual period). In some areas, group counselling resulted in more compliance rate as participants started discussing among themselves about the procedure and the importance of the cervical screening. Subsequent camps held in the same area led to more participation due to spread of information by word-of-mouth in their respective rural areas about the testing methods; hardly any dropouts were seen after this pre-test counselling. It was clear that it was possible to organize these camps owing to the co-operation from the staff at the local healthcare and community centres; this included the other voluntary organizations with whom we worked in order to conduct these camps. The local centres are now able to refer women who require screening to the city labs or alternately contact us for patient follow-up in case of abnormal paps.

Nutrition and immunity play a role in controlling cervix cancer, malnutrition leads to decreased immunity, giving the scope for the infectious organisms like bacteria, protozoa and fungi (Candida, Trichomonas, and Gardnerella) to harbor themselves in tissues altering the cervical microenvironment. Organisms like Trichomonas vaginalis and Gardnerella vaginalis are anaerobic bacteria, presence of these bacteria in the cervix increases the production of polyamines, which interact with organic acids of the cervix like succinic acid and acetic acid. This leads to cytotoxicity to vaginal cells and results in vaginal discharge. ${ }^{12}$ Infections in such a setting can lead to persistent HPV infection in the cervical area, eventually leading to carcinogenesis. These infections if identified by conventional pap test, can be eradicated by giving appropriate treatment like metronidazole or other antibiotics and prevent subsequent HPV persistence in the cervix tissue. In our study, most of our HPV positive cases were seen to have bacterial or candida infection. Trichomonas infection was seen in two cases of HIV. These findings are mentioned to draw attention to the necessity for proper care in sampling and handling of patient material by the personnel participating in these type of studies. Bacterial vaginosis is known to increases the nitrosamine content at the cervix and increase the DNA damage. This may change the cytokine (like interleukin 1b) profile, leading to compromised immunity. These cases can be treated appropriately before they turn cancerous. Atypical glandular cells can develop into adenocarcinomas, (these cases seemed to show HPV negativity) which is again why a cytological test is important.

Different cytologies observed in Pap smear included normal, inflammatory, infection, ASCUS, LSIL, HSIL and Squamous Cell Carcinomas (SCC). $30 \%$ of cases of cervical cancer are missed due to errors interpreting results of pap smears. So the presence of a cytopathologist who specializes in Pap smear evaluation becomes mandatory and would be preferable at a subsidized cost.

VIA, a simple technique doesn't require a laboratory to do the procedure, minimal training of the local health workers is sufficient. VIA procedure can itself reduce the burden of cervical cancer by regular visual inspection of the cervix. ${ }^{6}$ However, it is not utilized by many centres doing Pap testing despite the simple method of testing, due to certain percentage of false positivity in the observations. Nevertheless, for a generalized screening program especially in centres with inadequate infrastructural 
and laboratory support, this method definitely scores by its merits. ${ }^{8}$ In our camps, VIA initially conducted on the patients correlated well with the pap cytology results however we did not continue this for all the samples. In a previous hospital-based study from our group also, the same result was demonstrated. ${ }^{14}$

A strong etiological agent for cervical cancer is HPV (Human papilloma Virus). Most HPV infections regress spontaneously. Pap cytology along with HPV co-testing will give us better diagnosis. HPV testing as a screening tool gives us the presence of high-risk HPV prevalence (oncogenic HPV) in our population. It is important to do the follow-up of abnormal cytology and HPV DNA positive cases to decrease the risk of developing malignancy in the cervical tissue. To improve follow-up in abnormal cases not just in rural areas, but also in urban areas there is a need of post-test counselling. As a part of this, depending on visual examination, cytology and hr-HPV DNA test we counseled the patients about the disease, consequences due to lack of follow-up and further check-ups in routine screening for future. In rural areas where people cannot afford the cost of HPV testing, there is need to improve the technology for developing cost effective hr-HPV testing to reduce burden of this disease on the population.

Lack of awareness about the cervix screening procedures as compared to screening for other disease conditions is rampant in our part of the world and no educational programs have been chalked for reaching out to women belonging to different social background. In the Indian context, generally women ignore their health issues since they are busy with their families, especially working women at urban areas. Added to this, in some parts there is also low cooperation from the family with regard to issues pertaining to a women's health. Women should be coached to be proactive to manage their reproductive health and prevent factors within their reach, that are known contributory factors to cervical disease including cancer. ${ }^{15}$

\section{CONCLUSION}

We did limited screening for women (530 samples) and have given pap and HPV reports free of cost, however due to the lack of sufficient funds we could not continue this program for mass screening. Our study revealed a very high percentage of women who had never undergone cervical examination. Hence creating awareness became our primary focus point. Initiating national screening programs once or twice a year can decrease the burden of this disease which will make a great difference to the lives of the underprivileged and protect the family structure from getting disrupted due to the burden of a costly disease like cancer. We could approach this as national wide screening programs by training Medical Officers at local health care centres on cervical cancer screening procedure and follow-up especially in rural areas. Visual examination of the cervix combined with a simple VIA test (Visual inspection with acetic acid) can be undertaken for screening at low resource centres. In case of availability of a cytologist, a conventional pap test in the women with sample collected from the areas detected as abnormal by VIA may be effective in picking up an early lesion or tissue change with risk for cancer. There is a need to screen women for high risk HPV sub-types for successful application of any future vaccinations programs. The support of clinicians, pathologists and families will be key to the success of such screening programs aimed at improving women's health in developing countries like India.

\section{ACKNOWLEDGEMENT}

We acknowledge the financial support from the Department of Science and Technology (DST), New Delhi, Project no: [SR/FT/LS-106/2009(G)] for supporting our research. We are thankful to all patients and participants of the screening programs.

\section{CONFLICT OF INTEREST}

The authors declare no conflict of interest.

\section{ABBREVIATIONS}

HPV: Human Papilloma Virus; Hr-HPV: High risk Human Papilloma Virus; VIA: Visual Inspection with Acetic acid; DNA: Deoxyribonucleic Acid; PCR: Polymerase Chain Reaction; HIV: Human Immunodeficiency Virus; ASCUS: Atypical Squamous Cells of Undetermined Significance; LSIL: low grade intra-epithelial lesion; HSIL: High grade intraepithelial lesion; SCC: Squamous Cell Carcinoma.

\section{SUMMARY}

Despite its being the cause for a high mortality rate, cervical cancer screening is yet to be a part of any national screening exercise for women in India. Added to this is the lack of proper infrastructural facilities for screening at local centres and the required expertise for sampling and testing. Lack of awareness in women about the preventability of this cancer and hence the necessity for undergoing screening can be overcome largely, by sufficient interaction with local healthcare personnel and the women attending camps directly, thus improving compliance for testing. Improving facilities for high-risk HPV detection and regular Pap testing can go a long way in completely preventing this particular cancer afflicting women worldwide.

\section{REFERENCES}

1. Ferlay J, Soerjomataram I, Ervik M, Dikshit R, Eser S, Mathers C, et al. Cancer Incidence and Mortality Worldwide: IARC Cancer Base No. 11.; 2013.

2. National Centre for Disease Informatics Research, National Cancer Registry Programme, ICMR Time Trends in Cancer Incidence Rates, 1982-2010 Bangalore, India: NCDIR-NCRP (ICMR). 2013.

3. Swaminathan R, Rama R, Shanta V. Lack of active follow-up of cancer patients in Chennai, India: implications for population-based survival estimates. Bull World Health Organ. 2008;86(7):509-15.

4. Dutta S, Begum R, Mazumderlndra D, Mandal SS, Mondal R, Biswas J, et al. Prevalence of Human Papillomavirus in Women without Cervical Cancer: A Population-based Study in Eastern India. Int J Gynecol Pathol. 2012;31(2):178-83.

5. Goodman A, Nour N. Cervical Cancer Screening: The Complex Interplay of Medical Infrastructure, Society and Culture. Oncologist. 2014;19(4):315-7.

6. Shastri SS, Mittra I, Mishra GA Gupta S, Dikshit R, Singh S, et al. Effect of VIA Screening by Primary Health Workers: Randomized Controlled Study in Mumbai, India. J Natl Cancer Inst. 2014;106(3):dju009.

7. Aswathy S, Quereshi MA, Kurian B, Leelamoni K. Cervical cancer screening: Current knowledge and practice among women in a rural population of Kerala, India. Indian J Med Res. 2012;136(2):205-10.

8. Munoz N, Bosch XF, Sanjose SD, Herrero R, Castellsague X, Shah KV, et al Epidemiologic Classification of Human Papillomavirus Types Associated with Cervical Cancer. N Engl J Med. 2003;348(6):518-27.

9. Danaei G, Hoorn SV, Lopez AD, Murray CJL, Ezzati M. Causes of cancer in the world: comparative risk assessment of nine behavioural and environmental risk factors. Lancet. 2005;366(9499):1784-93.

10. McNicol P, Paraskevas M, Guijon F. Variability of polymerase chain reactionbased detection of human papillomavirus DNA is associated with the composition of vaginal microbial flora. J Med Virol. 1994;43(2):194-200.

11. Biswal BM, Singh KKB, Ismail MB, Jalal MIBA, Safruddin EISBE. Current Concept of Bacterial Vaginosis in Cervical Cancer. J Clin Gynecol Obstet. 2014;3(1):1-7

12. Goldie SJ, Gaffikin L, Jeremy D, Goldhaber FAB, Gordillo TA, Levin C, et al. Cost-Effectiveness of Cervical-Cancer Screening in Five Developing Countries. N Engl J Med. 2005;353(20):2158-68.

13. Sreedevi A, Javed R, Dinesh A. Epidemiology of cervical cancer with special focus on India. Int J Womens Health. 2015;7:405-14

14. Upendram P, Sahni S, Mohiuddin K, Poornima S, Gourishankar B, Vattam KK et al. Amplification of specific chromosomal regions assessed by fluorescent in situ hybridization on Pap smears to be added as screening tool for identifying women at risk of progressing to cervical cancer. Tumour Biol. 2017;39(10).

15. Siddharthar J, Rajkumar B, Deivasigamani K. Knowledge, Awareness and Prevention of Cervical Cancer among Women Attending a Tertiary Care Hospita in Puducherry, India. J Clin Diagn Res. 2014;6:8(6):OC01-3. 